\title{
Segmentation of MR Images of the Human Brain Using Fuzzy Adaptive Radial Basis Function Neural Network
}

\author{
J.K. Sing ${ }^{*}$, D.K. Basu, M. Nasipuri, and M. Kundu \\ Department of Computer Science \& Engineering, Jadavpur University, \\ Kolkata 700 032, India \\ jksing@ieee.org
}

\begin{abstract}
A method for segmentation of magnetic resonance (MR) images of the human brain using a fuzzy adaptive radial basis function neural network (FARBF-NN) has been proposed. Since the quality of MR images always gets affected by intensity in-homogeneities (artifacts or noises), generated due to the non-uniformity of magnetic fields during the acquisition process, thereby making segmentation task more difficult. The outputs of the hidden layer neurons of the FARBF-NN have been modified using a fuzzy membership function to eliminate the effect of noises present in the input image. The proposed method has been tested both on simulated and real patient MR brain images for segmentation and found to be better than the k-means clustering algorithm, the fuzzy cmeans (FCM) clustering algorithm, and the RBF neural network that uses kmeans clustering algorithm to select the centers of the RBFs in the hidden layer, in most of the cases.
\end{abstract}

\section{Introduction}

Image segmentation is a technique, which partitions a given image into a set of meaningful non-overlapping sub-regions, which are uniform with respect to certain characteristics, such as distribution of gray level or texture. In most of the cases, noises creep into the sampled images during the acquisition of medical images, thereby, making segmentation a difficult task. A number of methodologies, based on histogram analysis, region growing, edge detection, and pixel classification, have been proposed in the past. A survey of different segmentation techniques for MR images by Clarke et al. [1] gives many techniques for image processing.

In this work, to reduce the influence of noises, the output of each hidden layer neuron has been fuzzified (diluted / concentrated) by a fuzzy membership function. This has been done for each input feature vector depending on its position from the center of a radial basis function.

\section{Design of the FARBF Neural Network}

The architecture of an RBF-NN is shown in Fig.1. The role of an RBF neural network can be viewed as a function, which maps a feature vector $\mathbf{x}_{i}$ from p-dimensional feature space to c-dimensional decision space as $\mathrm{z}_{\mathrm{i}}$.

\footnotetext{
* Corresponding author.
} 


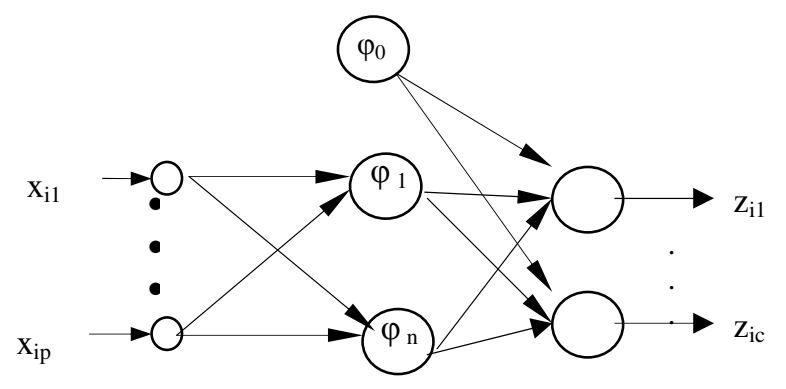

Fig. 1. Standard Radial Basis Function (RBF) Neural Network

The output of the $\mathrm{j}^{\text {th }}$ hidden layer neuron for $\mathrm{i}^{\text {th }}$ input pattern $\mathbf{x}_{\mathrm{i}}$ is generated by a Gaussian radial-basis function, which is defined as follows:

$$
\varphi_{j}\left(x_{i}\right)=\exp \left(-\frac{\left\|x_{i}-t_{j}\right\|^{2}}{2 \sigma_{j}^{2}}\right), j=1,2, \ldots, n ; i=1,2, \ldots, N
$$

where $t_{j}$ and $\sigma_{j}$ are the center and the width of the receptive field, respectively of the $\mathrm{j}^{\text {th }}$ neuron of the hidden layer, $\mathrm{n}$ and $\mathrm{N}$ are the total number of hidden layer neurons and total number of input patterns, respectively.

To make the RBF-NN fuzzy adaptive, $\varphi_{\mathrm{j}}\left(\mathrm{x}_{\mathrm{i}}\right)$ has been diluted (increased) or concentrated (decreased) by a fuzzy membership function, which is defined as follows:

$$
\begin{aligned}
& \text { If }\left(\varphi_{j}\left(x_{i}\right)\right)<0.5 \text { then } \\
& \qquad y_{j}\left(x_{i}\right)=\left(\varphi_{j}\left(x_{i}\right)\right)^{r} \\
& \text { else } \quad y_{j}\left(x_{i}\right)=\left(\varphi_{j}\left(x_{i}\right)\right)^{1 / r}
\end{aligned}
$$

where $r(r>0)$ defines the degree of fuzziness imposed on the output of hidden layer neurons and its value has been selected experimentally for which minimum mean square error (MSE) is achieved in the output layer during the training period. Therefore, the output of the $\mathrm{k}^{\text {th }}$ output layer neuron has been defined as follows:

$$
z_{i k}=\sum_{j=1}^{n}\left[y_{j}\left(x_{i}\right) w_{k j}+b_{k} w_{k}\right] k=1,2, \ldots, c, i=1,2, \ldots, N
$$

where $\mathrm{w}_{\mathrm{kj}}$ is the weight between the $\mathrm{j}^{\text {th }}$ neuron of the hidden layer and the $\mathrm{k}^{\text {th }}$ neuron of the output layer, $\mathrm{b}_{\mathrm{k}}$ and $\mathrm{w}_{\mathrm{k}}$ are unit positive bias and weight to the $\mathrm{k}^{\text {th }}$ output neuron from the bias neuron, respectively.

The centers of the different RBFs (hidden layer neurons) are selected by using a modified version of the k-means algorithm [2]. The width $\sigma_{j}(j=1,2, \ldots, n)$ of the receptive field of the hidden layer neuron is defined as follows:

$$
\sigma_{\mathrm{j}}=\beta \times \min \left\|\mathrm{t}_{\mathrm{j}}-\mathrm{t}_{\mathrm{i}}\right\|, \mathrm{i}, \mathrm{j}=1,2, \ldots, \mathrm{n} \text { and } \mathrm{i} \neq \mathrm{j}
$$

where $\beta(>1)$ is a constant and its value has been determined experimentally.

The weights of the links that connect hidden layer with output layer are estimated by using least mean square (LMS) algorithm. 


\section{Results and Discussion}

Performances of the proposed FARBF-NN, the RBF neural network using k-means algorithm, and the two frequently used methods for segmentation i.e., the k-means and the FCM have been compared on simulated and real patient MR images of the human brain. Simulated MR images of human brain are obtained from the McConnel Brain Imaging Centre of the Montreal Neurological Institute, McGill University. The MR images are segmented into four regions, corresponding to background and three tissue classes namely, gray matter (GM), white matter (WM), and cerebrospinal fluid (CSF). The proposed FARBF-NN uses seven hidden layer neurons to segment MR images. The learning rate parameter $(\eta)$ is taken as 0.002 in all the experiments.

\subsection{Quantitative Evaluation of Performance on Simulated Data}

Table 1 summarizes the error measures obtained by applying the FCM, k-means, RBF neural network using k-means algorithm, and the proposed FARBF-NN on a simulated T1-weighted image data, which is shown in Fig. 2(a). This error measure is the misclassification rate (MCR), which is defined as the number of pixels misclassified by the algorithm divided by the total number of pixels in the image.

Table 1. Error measure (\%) for T1-weighted simulated MR brain image

\begin{tabular}{|c|c|c|c|c|}
\hline Method & CSF & WM & GM & Average error \\
\hline FCM & 2.897 & 6.148 & 2.404 & 3.816 \\
\hline k-means & 2.891 & 6.160 & 2.320 & 3.790 \\
\hline RBF-NN +k-means & 5.024 & 7.974 & 1.827 & 4.942 \\
\hline Proposed FARBF-NN & 4.267 & 3.972 & 1.827 & 3.355 \\
\hline
\end{tabular}

Table 2. Error measure (\%) for T2-weighted simulated MR brain image

\begin{tabular}{|c|c|c|c|c|}
\hline Method & CSF & WM & GM & Average error \\
\hline FCM & 2.362 & 7.962 & 2.338 & 4.221 \\
\hline k-means & 2.320 & 7.890 & 2.350 & 4.186 \\
\hline RBF-NN + k-means & 3.606 & 5.114 & 2.614 & 3.778 \\
\hline Proposed FARBF-NN & 3.047 & 7.680 & 1.256 & 3.994 \\
\hline
\end{tabular}

We have also compared the performances of the above four methods on simulated T2-weighted brain images. The error measures obtained by the four methods are shown in table 2 .

\subsection{Visual Comparison of Performance}

Fig. 2 shows the segmentation results of the FARBF-NN, the RBF neural network using k-means algorithm, the k-means, and the FCM methods on a T1-weighted simulated normal MR brain image. It shows that the performance of the proposed method is better than its counterpart, which uses conventional k-means algorithm. Fig. 3 
shows the segmentation results by the above four methods on a T2-weighted real patient MR brain image. The noises have been removed from WM region of Fig. 3(a) by the proposed method, as shown in Fig. 3(b), while other methods fail to remove these noises.

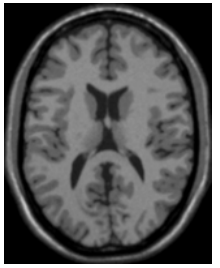

(a)

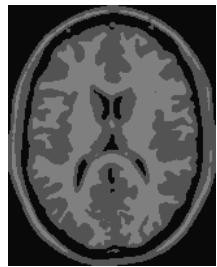

(b)

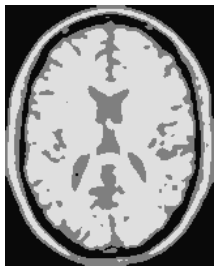

(c)

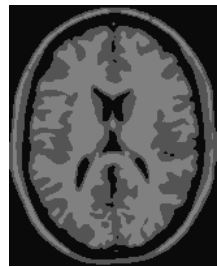

(d)

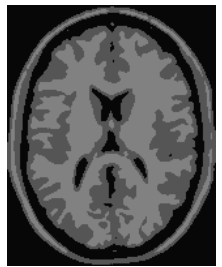

(e)

Fig. 2. Comparison of segmentation results. (a) The T1-weighted simulated MR image, and the (b), (c), (d), and (e) are the segmented images obtained by the proposed FARBF-NN, RBF neural network using k-means algorithm, k-means, and FCM, respectively.

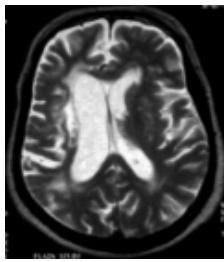

(a)

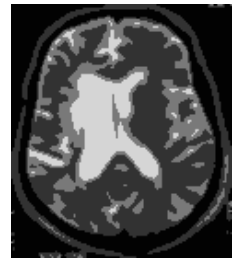

(b)

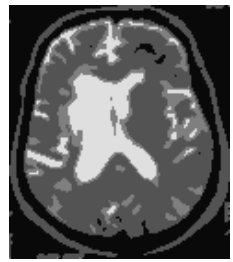

(c)

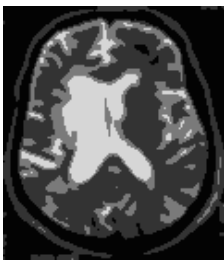

(d)

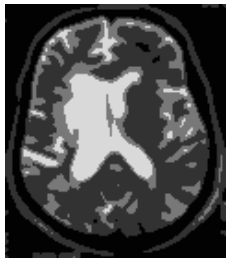

(e)

Fig. 3. Segmentation results. (a) The T2-weighted real patient MR brain image, and the (b), (c), (d), and (e) are the segmented images obtained by the proposed FARBF-NN, the RBF neural network using k-means algorithm, the k-means, and the FCM, respectively.

\section{Conclusion}

In this paper, an approach using a fuzzy adaptive RBF neural network has been proposed for MR brain image segmentation. The output of each hidden layer neuron has been fuzzified (diluted / concentrated) by a fuzzy membership function to reduce the effects of noises present in the MR image. The results of the segmentation have indicated its effectiveness in comparison to the other methods.

\section{Acknowledgement}

This work is partially supported by CMATER, SRUVM, and ADTUMI projects of the CSE Dept., Jadavpur University, Kolkata 700032. The authors are also thankful to Dr. P. K. Gharai and Dr. T. Dhibar of the Dept. of Neuroradiology, Bangur Institute of Neurology, Kolkata, India, for their invaluable discussion, suggestions and comments on the results of the segmentation of the MR brain images. 


\section{References}

1. L. P. Clarke et. al: MRI Segmentation: Methods and applications, Magn. Reson. Imag., (1995) 343-368

2. J. K. Sing, D. K. Basu, M. Nasipuri and M. Kundu: Center Selection of RBF Neural Network Based on Modified K-Means Algorithm With Point Symmetry Distance Measure, Foundation of Computing and Decision Sciences, (2004) 247-266 